\title{
A new multistage Parker-Sochacki method for solving the Troesch's problem
}

\author{
Saheed O. Akindeinde ${ }^{1 *}$, Samuel O. Adesanya ${ }^{2}$, Ramosheuw S. Lebelo ${ }^{3}$ and Kholeka C. Moloi ${ }^{4}$ \\ ${ }^{1}$ Department of Mathematics, Obafemi Awolowo University, Ile-Ife, Nigeria \\ ${ }^{2}$ Department of Mathematical Sciences, Redeemer's University, Ede, Nigeria \\ ${ }^{3}$ Faculty of Human Sciences, Vaal University of Technology, South Africa \\ ${ }^{4}$ Faculty of Human Sciences, Vaal University of Technology, South Africa \\ *Corresponding author E-mail:saheed.akindeinde@gmail.com, soakindeinde@oauife.edu.ng
}

\begin{abstract}
In this article, we introduce a new method to obtain an approximate analytical solution of the highly unstable Troesch's problem. In the proposed method, without recourse to any hyperbolic tangent transformation or finite term approximation of the hyperbolic sine function, the problem is recast as a system of projectively polynomials which allows straightforward computation of the series solution of the problem. The radius of convergence $R$ of the series solution to the problem is derived a-priorly in terms of the parameters of the polynomial system. Using a step length $h<R$, the problem domain is divided into subintervals, where corresponding subproblems are defined and solved with Parker-Sochacki method with very high accuracy. Highly accurate piecewise continuous approximate solution is thus obtained on the entire integration interval. The obtained solution, which is valid for every choice of the Troesch parameter $\lambda$, showed comparable accuracy to known numerical solutions in the literature. In particular, new results are presented for large values of $\lambda$ in the range $[20,500]$.
\end{abstract}

Keywords: Approximate analytical technique; Boundary value problems; Parker-Sochacki method; Troesch problem

\section{Introduction}

The Troesch's problem is a nonlinear two-point boundary value problem given by

$y^{\prime \prime}=\lambda \sinh \lambda y, \quad y(0)=0, y(1)=1$.

The problem arises in the study of confinement of a plasma column by a radiation pressure [1], and in the theory of gas porous electrodes $[2,3]$. The known exact solution to the Troesch problem is given implicitly by Roberts and Shipman in [4] as

$y(x)=\frac{2}{\lambda} \sinh ^{-1}\left[\frac{y^{\prime}(0)}{2} s c\left(\lambda x \mid 1-\frac{1}{4}\left(y^{\prime}(0)\right)^{2}\right)\right]$

where

with $m$ satisfying

$s c(\lambda \mid m)=\frac{\sinh \left(\frac{\lambda}{2}\right)}{\sqrt{1-m}}$

and $s c(\lambda \mid m)=\tan \phi$ denotes the Jacobi elliptic function. The parameters $\lambda, m$ and $\phi$ are related through the integral equation

$$
\lambda=\int_{0}^{\phi} \frac{1}{\sqrt{1-m \sin ^{2} \theta}} d \theta
$$

It has been established in [4] that the Troesch's BVP has a singularity located at approximately

$$
x_{s}=\frac{1}{\lambda} \ln \left(\frac{8}{y^{\prime}(0)}\right)
$$


This singularity lies within the interval of integration $[0,1]$ whenever $y^{\prime}(0)>8 e^{-\lambda}$ making numerical approximation a challenging task [5]. The sensitivity of the solution to the Troesch's parameter $\lambda$ in this way makes the problem a research attention in the literature and different methods have been proposed to derive approximate analytical and numerical solution to the problem. These methods include the Variational Iteration Method (VIM) [6], Adomian Decomposition Method (ADM) [7, 8], Homotopy Analysis Method (HAM) [9], Laplace Transform Method [10], Homotopy Perturbation Method (HPM) [11] and their various modifications. In general the aforementioned methods belong to either of the following two categories:

1. Methods involving the transformation $y(x)=(4 / \lambda) \tanh ^{-1}(u(x))$ where $u(x)$ is a solution to a strongly nonlinear BVP;

2. Methods involving the truncation or approximation of the hyperbolic sinh .

In the second category the function sinh is replaced by only a few terms of its Taylor expansion. While clearly only approximated problem is being solved in the second case, concerns have also been raised on the validity of the transformation $y(x)=(4 / \lambda) \tanh ^{-1}(u(x))$ for large values of $\lambda$.

In this article, we propose a new method to derive approximate analytical solution to the Troesch's problem in form of a piecewise continuous function. We emphasize that our method involves neither the hyperbolic tangent transformation nor truncation of Taylor's expansion of the hyperbolic sine function. Here, by using appropriate auxiliary variables, the problem is recast as a system of projectively polynomial ordinary differential equations which allows the coefficients of the series solution to be computed recursively via the Parker-Sochacki method. Both the solution function $y(x)$ and its first derivative $y^{\prime}(x)$ are computed simultaneously.

To extend the accuracy of the obtained series solution, we divide the problem's domain into subintervals so that the corresponding subproblems are defined and are amenable to the Parker-Sochacki method with very high accuracy. The domain split technique is popular; It has been used to improve the accuracy of Adomian Decomposition method [12, 13], the Homotopy Analysis Method [14, 15], the Variational Analysis Method [16], Homotopy Perturbation Method [17] and Differential Transformation Method [18]. However, major drawback of these methods is that as subintervals becomes more, the computation becomes more tedious. The tradeoff between the intended accuracy and resulting computational complexity becomes undesirable. However, in this work, we leverage on the computational simplicity of the Parker-Sochacki to devise a multi-interval technique for the solution of the Troesch's problem. The proposed method is highly efficient both in terms of solution accuracy and decreased computational complexities.

\section{The Multistage Parker-Sochacki Method}

Here, the basics of the proposed method are presented. We describe the conventional Parker-Sochacki method and introduce the domain split technique of the new approach.

\subsection{The Parker-Sochacki Method (PSM) and Projectively polynomials}

To effectively describe the Parker-Sochacki Method (PSM), the following preliminaries are essential.

Definition 2.1. [19] (Polynomial Systems) $G: \mathbb{R}^{d} \rightarrow \mathbb{R}^{d}$ is an $\mathbb{R}^{d}$-polynomial if each component of $G$ is a polynomial function on $\mathbb{R}^{d}$. A polynomial system is an autonomous initial value system of differential equations of the form

$y^{\prime}(x)=G(y(x)), \quad x_{0} \leq x \leq x_{f}, \quad y\left(x_{0}\right)=y_{0}$

for some polynomial function $G: \mathbb{R}^{d} \rightarrow \mathbb{R}^{d}, x_{0}, x_{f} \in \mathbb{R}^{d}, y_{0} \in \mathbb{R}^{d}$.

Definition 2.2. (Projectively Polynomials) Let $\mathscr{A}$ be the set of real-analytic functions. An element $f$ of $\mathscr{A}$ is said to be projectively polynomial if $f$ is a component of the solution to a polynomial system, that is, for some d there is a polynomial $G$ in $d+1$ variables so that $G\left(u, u^{\prime}, \ldots, u^{(d)}\right)=0$.

Let us illustrate polynomial projection with the following example. Consider a non-autonomous, non-polynomial system of equations reported in [20]

$y_{1}^{\prime}=\frac{1}{2} \frac{y_{1}}{t+1}-2 t y_{2}, \quad y_{1}(0)=1$,

$y_{2}^{\prime}=\frac{1}{2} \frac{y_{2}}{t+1}+2 t y_{1}, \quad y_{2}(0)=0$.

By introducing auxilliary variables $y_{3}=t, y_{4}=1 /(t+1)$ the solutions $y_{1}, y_{2}$ to the system are now equivalent to the solutions $y_{1}, y_{2}$ of the autonomous polynomial system

$y_{1}^{\prime}=\frac{1}{2} y_{1} y_{4}-2 y_{3} y_{2}, \quad y_{1}(0)=1$,

$y_{2}^{\prime}=\frac{1}{2} y_{2} y_{4}+2 y_{3} y_{1}, \quad y_{2}(0)=0$,

$y_{3}^{\prime}=1, \quad y_{3}(0)=0$,

$y_{4}^{\prime}=-y_{4}^{2}, \quad y_{4}(0)=1$.

Based on polynomial projection technique, Parker and Sochacki in [21] and [22] modified the Picard iteration to compute power series solution to the first order non-autonomous system of the form

$\mathbf{y}^{\prime}=f(x, \mathbf{y}), \quad x_{0} \leq x \leq x_{f}, \quad \mathbf{y}\left(x_{0}\right)=\mathbf{y}_{0}$. 
Let us now briefly summarise the method. In the first step, using appropriate auxiliary variables, the above problem is recast as a polynomial system of the form (4), namely

$\mathbf{y}^{\prime}=f(\mathbf{y}), \quad x_{0} \leq x \leq x_{f}, \quad \mathbf{y}\left(x_{0}\right)=\mathbf{y}_{0}$.

Here, the function $f$ is a vector of polynomials, that is, $f: \mathbb{R}^{d} \rightarrow \mathbb{R}^{d}$ where $d$ is the dimension of the polynomial system. For the moment, let us assume that $x_{0}=0$. Secondly, we assume Maclaurin series expansion for the function $\mathbf{y}(x)$ as

$\mathbf{y}(x)=\sum_{i=0}^{N} \mathbf{y}_{i} x^{i}$

where the coefficients $\mathbf{y}_{0}=\mathbf{y}(0), \mathbf{y}_{1}=\mathbf{y}^{\prime}(0), \mathbf{y}_{2}=\frac{1}{2 !} \mathbf{y}^{\prime \prime}(0), \ldots$ are to be determined. Since the function $f$ is a polynomial function of $y$, we can similarly write

$$
f(\mathbf{y}(x))=\sum_{i=0}^{N} f_{i} x^{i}
$$

Furthermore, observe that on differentiating (13) with respect to $x$ and shifting index appropriately we have

$\mathbf{y}^{\prime}(x)=\sum_{i=0}^{N}(i+1) \mathbf{y}_{i+1} x^{i}$

so that from (12), we get

$\mathbf{y}_{i+1}=\frac{f_{i}}{i+1}$

where the coefficients $f_{i}$ are computed by repeated use of Cauchy product [23] e.g. if $f(y)=y^{2}$, then $f_{i}=\sum_{j=0}^{i} y_{j} y_{i-j}$. The obtained coefficients $y_{i+1}$ above are then used in (13) to obtain the series solution of the problem (11) as

$$
\mathbf{y}(x)=y_{0}+\sum_{i=1}^{N} \frac{f_{i-1}}{i} x^{i}
$$

Now in the case $x_{0} \neq 0$, the series solution is computed normally as described above. Let this series solution be denoted by $\phi(t)$. The solution to the original problem is then

$y(x)=\phi\left(x-x_{0}\right)=y\left(x_{0}\right)+\sum_{i=1}^{N} \frac{f_{i-1}}{i}\left(x-x_{0}\right)^{i}$.

As can be seen above, the PSM is very easy to implement. This makes the method applicable to a wide range of differential equations that can be written in the form (12) including strongly nonlinear coupled differential equations.

\subsection{Domain Split Technique}

The validity of power series solution to a differential equation are often restricted to small intervals. To improve the usefulness of the series solution computed via the method described above, we consider the problem on smaller subintervals where high accuracy of the method is assured. In the new approach, the domain of the problem $\left[x_{0}, x_{f}\right]$ is divided into non-overlapping subintervals $I_{k}=\left[x_{k-1}, x_{k-1}+h\right], k=$ $1,2,3, \ldots, n$ such that $\cup\left[x_{k-1}, x_{k-1}+h\right]=\left[x_{0}, x_{f}\right]$. Here $h$ denotes a fixed step-size which is related to the number of subintervals $n$ through $h=\frac{x_{f}-x_{0}}{n}$. On the first subinterval $I_{1}=\left[x_{0}, x_{0}+h\right]$, the problem is solved using the Parker-Sochacki method to obtain

$y_{1}(x)=y\left(x_{0}\right)+\sum_{i=1}^{N} \frac{f_{i-1}}{i}\left(x-x_{0}\right)^{i}$.

In the next subinterval $I_{2}=\left[x_{0}+h, x_{0}+2 h\right]$, the numerical value of $y_{1}$ at the end of the first interval $x_{0}+h$ becomes the initial condition for the problem on $I_{2}$ i.e.

$\left(y_{2}\right)_{0}=y_{1}\left(x_{0}+h\right)$.

In general, function evaluation of the solution at the end of one interval becomes the initial conditions for the next subinterval. Therefore, on the $k$ th subinterval $I_{k}$, the problem to be solved is given by

$y_{k}^{\prime}=f\left(y_{k}\right), \quad t \in\left[x_{k-1}, x_{k-1}+h\right], \quad\left(y_{k}\right)_{0}=y_{k-1}\left(x_{0}+(k-1) h\right)$.

Continuing this process, the problem is solved from one subinterval to the next to obtain a convergent piecewise continuous approximation to the solution of the problem. It should be noted that the advantage of Parker-Sochacki method is that it allows on-the-fly change of the order of approximation $N$. Therefore, besides reducing the length of the subintervals to achieve better results, the overall accuracy of the method can be improved by increasing the order of approximation in each subintervals. 


\subsection{A-priori Computation of Radius of Convergence}

The standard error bound for the Taylor series approximation of solution of (11) is given by

$\left\|y(x)-\sum_{i=0}^{N} y_{i} x^{i}\right\| \leq \frac{f^{(k)}(x, y(x))|x|^{N+1}}{(N+1) !}, \quad|x| \in\left[x_{0}, x_{f}\right]$

where $f^{(k)}$ denotes the $k$ th derivative of function $f$. One of the disadvantage of the error estimate above is the dependence of the estimate on a derivative of $f$ and the yet unknown solution $y$ of the original problem. Computing a bound for the derivative can be cumbersome even for relatively modest functions. Normally, an ideal error bound would be independent of derivatives of $f$ and easily be re-calculated for higher order approximations. Such a sharp error bound has been developed for polynomial system of the form (12) and reported below.

\subsubsection{Derivative - free Error Bound for Polynomial System}

In [20], the authors derived a derivative-free error bound for series solution of differential equations. In what follows, unless otherwise stated, we shall follow the notations used in [24]. The norm $\|\cdot\|$ is defined as

$\left\|\left(y_{1}, y_{2}, \ldots, y_{n}\right)\right\|=\max _{1 \leq i \leq n}\left|y_{i}\right|$

If $f: \mathbb{R}^{d} \rightarrow \mathbb{R}^{d}$ is a vector of polynomials and $Y^{\alpha}=y_{1}^{\alpha_{1}} y_{2}^{\alpha_{2}} \ldots y_{d}^{\alpha_{d}}$, we write

$f\left(Y_{1}, Y_{2}, \ldots, Y_{d}\right)=\sum_{|\alpha| \leq k} a_{\alpha} Y^{\alpha}$

where $k=\operatorname{deg}\left(p_{i}\right)$ is the degree of $p_{i}$ and $|\alpha|=\alpha_{1}+\alpha_{2}+\cdots+\alpha_{d}$. Furthermore, we define

$\Sigma p_{i}=\sum_{|\alpha| \leq k}\left|a_{\alpha, i}\right|$

$\operatorname{deg}(p)=\max \left\{\operatorname{deg}\left(p_{1}\right), \operatorname{deg}\left(p_{2}\right), \ldots, \operatorname{deg}\left(p_{d}\right)\right\}$ and $\Sigma p=\max \left\{\Sigma p_{1}, \Sigma p_{2}, \ldots, \Sigma p_{d}\right\}$.

Theorem 2.3. [20] If y satisfies (11), $k=\operatorname{deg}(p) \geq 2, \alpha=\max \left\{1,|| y_{0} \|\right\}, M=(k-1) \Sigma p \alpha^{k-1}, x_{0}=0$, and $|x|<\frac{1}{M}$ then

$\left\|y(x)-\sum_{i=0}^{N} y_{i} x^{i}\right\| \leq \frac{\alpha|M x|^{N+1}}{1-|M x|}$.

The advantage of the above error estimate over the standard Taylor series method is obvious; the estimate does not involve the derivative of function $f$ and the order $N$ and corresponding error estimate can be computed on-the-fly.

By design, the method proposed in this article requires that the initial data for subsequent subinterval be calculated from the truncated solution on current subinterval. Since the solution on current subinterval is only an approximation to the unknown solution on that subinterval, errors are propagated from one subinterval to the next. The following corollary gives the global variation of the error in the solution of (11) over a subinterval.

Corollary 2.4. [24] If y satisfies (11), $k=\operatorname{deg}(p) \geq 2, \alpha=\max \left\{1,\left\|y_{0}\right\|\right\}, M=(k-1) \Sigma p \alpha^{k-1}, x_{0}=0$, and $|x|<\frac{1}{M}$ then

$\left\|y(x)-y_{0}\right\| \leq \frac{\alpha|M x|}{1-|M x|}$

Remark 2.5. It follows from Theorem 2.3 and Corollary 2.4 that the smallest radius of convergence of series solution to problem (12) is $1 / M$ where $M$ is defined by parameters of the system (12), given in Theorem 2.3 above.

\section{Application to the Troesch's problem}

Here, the proposed method is applied to the Troesch's problem (1). The proposed method requires that the problem be re-written in the form $y^{\prime}=f(y)$ where $f$ is a vector of polynomial function. Therefore, the first step in solving the Troesch's problem using the proposed method is to recast the problem as a system of projectively polynomial functions. This projection is achieved by the auxiliary variables $y^{\prime}=u, v=\sinh \lambda y$ and $w=\cosh \lambda y$ so that

$y^{\prime}=u, \quad u^{\prime}=\lambda v, \quad v^{\prime}=\lambda u w, \quad w^{\prime}=\lambda u v$

with initial conditions

$y(0)=0, \quad u(0)=y^{\prime}(0), \quad v(0)=0, \quad w(0)=1$.

Clearly the nonlinearity $\sinh \lambda y(x)$ has been reduced to a polynomial-type nonlinearity. However, the approach here does not depend on a transformation whose validity is not guaranteed for larger value of the Troesch's parameter $\lambda$.

Now writing

$y=\sum_{i=0}^{N} y_{i} x^{i}, \quad u=\sum_{i=0}^{N} u_{i} x^{i}, \quad v=\sum_{i=0}^{N} v_{i} x^{i}, w=\sum_{i=0}^{N} w_{i} x^{i}$ 
where $N$ denotes the truncation point of the infinite series for each of the variables. Then in line with discussion of Section 2, the coefficients of the series solution above are given by

$y_{i+1}=\frac{u_{i}}{i+1}, \quad u_{i+1}=\lambda \frac{v_{i}}{i+1}, \quad v_{i+1}=\lambda \frac{\sum_{j=0}^{i} u_{i-j} w_{j}}{i+1}, \quad w_{i+1}=\lambda \frac{\sum_{j=0}^{i} u_{i-j} v_{j}}{i+1}, i=0,1,2, \ldots, N$.

These recurrence relations yield for even $i, y_{i}=0$ and for odd $i$

$y_{1}=y^{\prime}(0), \quad y_{3}=\frac{1}{3 !} \lambda^{2} y^{\prime}(0), \quad y_{5}=\frac{1}{5 !} \lambda^{4}\left(y^{\prime}(0)+y^{\prime}(0)^{3}\right), \quad y_{7}=\frac{1}{7 !} \lambda^{6}\left(y^{\prime}(0)+11 y^{\prime}(0)^{3}+y^{\prime}(0)^{5}\right)$,

$y_{9}=\frac{1}{9 !} \lambda^{8}\left(y^{\prime}(0)+102 y^{\prime}(0)^{3}+57 y^{\prime}(0)^{5}+y^{\prime}(0)^{7}\right)$,

$y_{11}=\frac{1}{11 !} \lambda^{10}\left(y^{\prime}(0)+922 y^{\prime}(0)^{3}+1923 y^{\prime}(0)^{5}+247 y^{\prime}(0)^{7}+y^{\prime}(0)^{9}\right)$,

$y_{13}=\frac{1}{13 !} \lambda^{12}\left(y^{\prime}(0)+8303 y^{\prime}(0)^{3}+54415 y^{\prime}(0)^{5}+24040 y^{\prime}(0)^{7}+1013 y^{\prime}(0)^{9}+y^{\prime}(0)^{11}\right)$, etc.

Hence, the series solution to the Troesch's problem is obtained as

$$
\begin{aligned}
y(x)= & y^{\prime}(0) x+\frac{1}{3 !} \lambda^{2} y^{\prime}(0) x^{3}+\frac{1}{5 !} \lambda^{4}\left(y^{\prime}(0)+y^{\prime}(0)^{3}\right) x^{5}+\frac{1}{7 !} \lambda^{6}\left(y^{\prime}(0)+11 y^{\prime}(0)^{3}+y^{\prime}(0)^{5}\right) x^{7} \\
& +\frac{1}{9 !} \lambda^{8}\left(y^{\prime}(0)+102 y^{\prime}(0)^{3}+57 y^{\prime}(0)^{5}+y^{\prime}(0)^{7}\right) x^{9} \\
& +\frac{1}{11 !} \lambda^{10}\left(y^{\prime}(0)+922 y^{\prime}(0)^{3}+1923 y^{\prime}(0)^{5}+247 y^{\prime}(0)^{7}+y^{\prime}(0)^{9}\right) x^{11} \\
& +\frac{1}{13 !} \lambda^{12}\left(y^{\prime}(0)+8303 y^{\prime}(0)^{3}+54415 y^{\prime}(0)^{5}+24040 y^{\prime}(0)^{7}+1013 y^{\prime}(0)^{9}+y^{\prime}(0)^{11}\right) x^{13}+\cdots
\end{aligned}
$$

\subsection{Convergence and Multistage Implementation}

A direct consequence of Theorem 2.3 and Corollary 2.4 is the assurance of a tight error bound for convergent series approximation by the conventional Parker-Sochacki method with radius of convergence $(-1 / M, 1 / M)$ where $M$ is as defined in Theorem 2.3. For the Troesch's problem and from (14) and (15), it is clear that $k=2, \alpha=\max \left(1,\left\|y_{0}\right\|\right), \Sigma p=\max (1, \lambda)$. Hence,

$M= \begin{cases}\alpha \lambda & \text { if } \lambda>1 \\ \alpha & \text { if } \lambda \leq 1\end{cases}$

Therefore, for $\lambda>1$, the series (17) is convergent in the interval $\left(-\frac{1}{\alpha \lambda}, \frac{1}{\alpha \lambda}\right)$ with guaranteed error bound $\frac{\left.|\lambda x|\right|^{N+1}}{1-|\lambda x|}$. As suggested in [20], the convergence of the series can be extended to any desired interval $[0, T]$ where $T>\frac{1}{\lambda}$ by stepping out to $T$ in a multistage fashion. For that purpose, we introduce the nodes $x_{i}=i h$ where $h$ is a fixed step-size and $i=0,1, \ldots, n=\frac{1}{h}$. In the first subinterval $[0, h]$ the approximate solution is given by (17). For the next subinterval $[h, 2 h]$, the initial condition $\left(y_{2}\right)_{0}$ is obtained by evaluating (17) at $h$, which is then used in the computation of new approximation $y_{2}(x)$ on $[h, 2 h]$. This procedure is repeated until the last subinterval $[(n-1) h, 1]$. It is worth mentioning here that by choosing the step-size $h$ conveniently small (smaller than the computed $1 / M$ on that subinterval e.g $1 / 2 M$ ), only few terms (which translates to few recursions) of the series solution are needed to ensure high accuracy of the approximation on the subinterval. More precisely, with $h=1 / 2 M$, any degree $N$ polynomial approximation satisfying

$N>\frac{-\ln (\varepsilon / \alpha)}{\ln (2)}$

ensures that the error at this level is less than $\varepsilon$, see [20].

Remark 3.1. Note that by definition, the parameter $\alpha$ in Theorem 2.3 depends on the initial conditions. Therefore, on each subintervals, as new initial conditions are computed, so is $\alpha$ and subsequently recalculation of the convergence parameter $M$. Hence, for $x \in(-1 / M, 1 / M)$, the error bound of Theorem 2.3 is guaranteed.

\subsection{Estimation of initial slope $y^{\prime}(0)$}

Let us now comment on the estimation of the missing data $y^{\prime}(0)$. This has been achieved in the literature through the shooting technique and its various modifications. The shooting technique requires that the problem be re-written as an initial value problem

$$
w^{\prime \prime}=\lambda \sinh \lambda w, \quad w(0)=0, w^{\prime}(0)=s
$$

where $w(x, s):=y(x)$. By enforcing the remaining boundary condition $y(1)=1$, the parameter $s$ is obtained iteratively using Newton method as a solution to the nonlinear function

$$
g(s)=w(1, s)-1 .
$$

However, as hinted earlier, the Troesch's problem is very sensitive to the choice of the shooting parameter $s$. It is well known [25] that the Troesch's problem has a pole at $x_{s}=\frac{1}{\lambda} \ln \left(\frac{8}{|s|}\right)$ which lies within the interval of integration. This make numerical integration impossible 
on the entire interval $[0,1]$ unless $s$ is chosen in such a way that the pole $x_{s}$ is located out of the integration interval. To achieve this, the parameter $s=y^{\prime}(0)$ must be chosen to satisfy the restrictive condition

$$
|s| \leq s^{*}=8 e^{-\lambda}
$$

For $\lambda=0.5$ and those in the range $\left[1,10^{12}\right]$, highly accurate values of $y^{\prime}(0)$ have been reported in the literature. The authors in [25] implemented both the simple and multiple shooting technique for the Troesch's problem and the values of $y^{\prime}(0)$ for the Troesch's parameter $0 \leq \lambda \leq 20$ were presented. In article [26], the authors gave a complete and accurate list of values for the parameter $s=y^{\prime}(0)$ in the range $1 \leq \lambda \leq 10^{12}$. For different values of the Troesch's parameter $\lambda$, these values of $y^{\prime}(0)$ are reported in Table 1. It is also well known [27] that for $\lambda>20$, the formula

is a good approximation of $y^{\prime}(0)$.

$$
y^{\prime}(0)=10^{-12} e^{29.71-\lambda}, \quad \lambda>20, \quad y^{\prime}(0)<10^{-4}
$$

\section{Results and discussions}

The proposed multistage method is implemented for the Troesch problem over the interval $[0,1]$. The interval is divided into subintervals using a fixed step-size $h$. On each subinterval, approximation (17) is used. We again emphasize that the proposed method does not depend on any hyperbolic tangent transformation and neither does it require truncation of the hyperbolic sine nonlinearity. Nevertheless, the computed results show high degree of accuracy compared to results obtained through other methods, see Section 4. Another advantage of the proposed method is that it allows change of order of approximation on the 'fly'. In other words, approximations of arbitrary order can be computed by changing the variable $N$ in the computer implementation.

Table 1: Initial slope $y^{\prime}(0)$ for different choices of the Troesch Parameter $\lambda$.

\begin{tabular}{cc}
\hline$\lambda$ & $y^{\prime}(0)$ \\
\hline 0.5 & $9.5904379541 \times 10^{-1}[28,29]$ \\
\hline 1 & $8.452026845 \times 10^{-1}[28,29]$ \\
\hline 5 & $4.575046433 \times 10^{-1}[26]$ \\
\hline 10 & $3.583377845 \times 10^{-4}[26]$ \\
\hline 15 & $2.444513025 \times 10^{-6}[26]$ \\
\hline 20 & $1.648773182 \times 10^{-8}[26]$ \\
\hline 25 & $1.111027228 \times 10^{-10}[26]$ \\
\hline 30 & $7.4891739203 \times 10^{-13}[5]$ \\
\hline 50 & $1.5433079539 \times 10^{-21}[5]$ \\
\hline 60 & $7.0074459344 \times 10^{-26}[5]$ \\
\hline 80 & $1.4448778857 \times 10^{-34}[5]$ \\
\hline 100 & $2.9798472766 \times 10^{-43}[5]$ \\
\hline 200 & $1.107117221 \times 10^{-86}[26]$ \\
\hline 500 & $5.699661125 \times 10^{-217}[26]$ \\
\hline $10^{3}$ & $4.060767116 \times 10^{-434}[26]$ \\
\hline $10^{4}$ & $9.083870920 \times 10^{-4343}[26]$ \\
\hline $10^{5}$ & $2.850359652 \times 10^{-43429}[26]$ \\
\hline $10^{6}$ & $2.637465182 \times 10^{-434294}[26]$ \\
\hline $10^{12}$ & $4.479838276 \times 10^{-434294481903}[26]$ \\
\hline
\end{tabular}

Table 2: Solution $y(x)$ for $\lambda=0.5, N=5, h=10^{-3}$.

\begin{tabular}{ccc}
\hline$x$ & $y(x)$ This article (Approximate Analytical) & Exact $[28,29]$ \\
\hline 0.1 & 0.0959443493 & 0.0959443493 \\
\hline 0.2 & 0.1921287477 & 0.1921287477 \\
\hline 0.3 & 0.2887944009 & 0.2887944009 \\
\hline 0.4 & 0.3861848464 & 0.3861848464 \\
\hline 0.5 & 0.4845471647 & 0.4845471647 \\
\hline 0.6 & 0.5841332484 & 0.5841332484 \\
\hline 0.7 & 0.6852011483 & 0.6852011483 \\
\hline 0.8 & 0.7880165226 & 0.7880165226 \\
\hline 0.9 & 0.8928542161 & 0.8928542161 \\
\hline
\end{tabular}


Table 3: Solution $y(x)$ for $\lambda=1, N=5, h=10^{-3}$.

\begin{tabular}{ccc}
\hline$x$ & $y(x)$ This article (Approximate Analytical) & Exact $[28,29]$ \\
\hline 0.1 & 0.0846612565 & 0.0846612565 \\
\hline 0.2 & 0.1701713580 & 0.1701713582 \\
\hline 0.3 & 0.2573939078 & 0.2573939080 \\
\hline 0.4 & 0.3472228548 & 0.3472228551 \\
\hline 0.5 & 0.4405998348 & 0.4405998351 \\
\hline 0.6 & 0.5385343976 & 0.5385343980 \\
\hline 0.7 & 0.6421286086 & 0.6421286091 \\
\hline 0.8 & 0.7526080933 & 0.7526080939 \\
\hline 0.9 & 0.8713625190 & 0.8713625196 \\
\hline
\end{tabular}

Table 4: Solution $y(x)$ for $\lambda=10, N=5, h=10^{-3}$.

\begin{tabular}{ccc}
\hline$x$ & $y(x)$ This article (Approximate Analytical) & $y(x)[26] \mathrm{HPM}$ \\
\hline 0.1 & $4.211189925699 \times 10^{-5}$ & $4.211189927237 \times 10^{-5}$ \\
\hline 0.2 & $1.299641157763 \times 10^{-4}$ & $1.299641158237 \times 10^{-4}$ \\
\hline 0.3 & $3.589784012586 \times 10^{-4}$ & $3.589784013896 \times 10^{-4}$ \\
\hline 0.4 & $9.779027714458 \times 10^{-4}$ & $9.779027718029 \times 10^{-4}$ \\
\hline 0.5 & $2.659020489380 \times 10^{-3}$ & $2.659020490351 \times 10^{-3}$ \\
\hline 0.6 & $7.228931212877 \times 10^{-3}$ & $7.228931212877 \times 10^{-3}$ \\
\hline 0.7 & $1.966406308982 \times 10^{-2}$ & $1.966406309701 \times 10^{-2}$ \\
\hline 0.8 & $5.373032933073 \times 10^{-2}$ & $5.373032935060 \times 10^{-2}$ \\
\hline 0.9 & $1.521140763436 \times 10^{-1}$ & $1.521140764047 \times 10^{-1}$ \\
\hline
\end{tabular}

Table 5: Solution $y(x)$ for $\lambda=30, N=5, h=10^{-4}$.

\begin{tabular}{ccc}
\hline$x$ & $y(x)$ This article (Approximate Analytical) & [5] (Numerical by Finite Difference) \\
\hline 0.1 & $2.5008535880 \times 10^{-13}$ & $2.5000565011 \times 10^{-13}$ \\
\hline 0.2 & $5.0355497250 \times 10^{-12}$ & $5.0339291116 \times 10^{-12}$ \\
\hline 0.3 & $1.0114233983 \times 10^{-10}$ & $1.0110947278 \times 10^{-10}$ \\
\hline 0.4 & $2.0314982320 \times 10^{-9}$ & $2.0308317333 \times 10^{-9}$ \\
\hline 0.5 & $4.0803732750 \times 10^{-8}$ & $4.0790218297 \times 10^{-8}$ \\
\hline 0.6 & $8.1956488076 \times 10^{-7}$ & $8.1929087544 \times 10^{-7}$ \\
\hline 0.7 & $1.6461400757 \times 10^{-5}$ & $1.6455845788 \times 10^{-5}$ \\
\hline 0.8 & $3.3063674875 \times 10^{-4}$ & $3.3052414064 \times 10^{-4}$ \\
\hline 0.9 & $6.6465028445 \times 10^{-3}$ & $6.6442145404 \times 10^{-3}$ \\
\hline 0.95 & $3.0272579081 \times 10^{-2}$ & $3.0261754073 \times 10^{-2}$ \\
\hline 0.97 & $5.7559304786 \times 10^{-2}$ & $5.7536749450 \times 10^{-2}$ \\
\hline 0.98 & $8.2266915709 \times 10^{-2}$ & $8.2230359053 \times 10^{-2}$ \\
\hline 0.99 & $1.2706204341 \times 10^{-1}$ & $1.2698617477 \times 10^{-1}$ \\
\hline
\end{tabular}

Table 6: Solution $y(x)$ for $\lambda=50, N=5, h=10^{-4}$.

\begin{tabular}{ccc}
\hline$x$ & $y(x)$ This article (Approximate Analytical) & $y(x)[5]$ (Numerical by Finite Difference) \\
\hline 0.1 & $2.2903681019 \times 10^{-21}$ & $2.2903150821 \times 10^{-21}$ \\
\hline 0.2 & $3.3993619789 \times 10^{-19}$ & $3.3992341127 \times 10^{-19}$ \\
\hline 0.3 & $5.0451005127 \times 10^{-17}$ & $5.0448377557 \times 10^{-17}$ \\
\hline 0.4 & $7.4875930508 \times 10^{-15}$ & $7.4870947647 \times 10^{-15}$ \\
\hline 0.5 & $1.1112573387 \times 10^{-12}$ & $1.1111673106 \times 10^{-12}$ \\
\hline 0.6 & $1.6492521222 \times 10^{-10}$ & $1.6490946502 \times 10^{-10}$ \\
\hline 0.7 & $2.4477071761 \times 10^{-8}$ & $2.4474380584 \times 10^{-8}$ \\
\hline 0.8 & $3.6327195481 \times 10^{-6}$ & $3.6322675919 \times 10^{-6}$ \\
\hline 0.9 & $5.3915154641 \times 10^{-4}$ & $5.3907666732 \times 10^{-4}$ \\
\hline 0.95 & $6.5829287458 \times 10^{-3}$ & $6.5819617827 \times 10^{-3}$ \\
\hline 0.97 & $1.8159580271 \times 10^{-2}$ & $1.8156763441 \times 10^{-2}$ \\
\hline 0.98 & $3.0884269307 \times 10^{-2}$ & $3.0879054743 \times 10^{-2}$ \\
\hline 0.99 & $5.6288492523 \times 10^{-2}$ & $5.6276053038 \times 10^{-2}$ \\
\hline
\end{tabular}


Table 7: Solution $y(x)$ for $\lambda=60, N=5, h=10^{-4}$.

\begin{tabular}{ccc}
\hline$x$ & $y(x)$ This article (Approximate Analytical) & $y(x)[5]$ (Numerical by Finite Difference) \\
\hline 0.1 & $2.3558234074 \times 10^{-25}$ & $2.3557350661 \times 10^{-25}$ \\
\hline 0.2 & $9.5041283444 \times 10^{-23}$ & $9.5035343622 \times 10^{-23}$ \\
\hline 0.3 & $3.8342390313 \times 10^{-20}$ & $3.8339035541 \times 10^{-20}$ \\
\hline 0.4 & $1.5468424264 \times 10^{-17}$ & $1.5466684184 \times 10^{-17}$ \\
\hline 0.5 & $6.2404077379 \times 10^{-15}$ & $6.2395497509 \times 10^{-15}$ \\
\hline 0.6 & $2.5175601646 \times 10^{-12}$ & $2.5171510991 \times 10^{-12}$ \\
\hline 0.7 & $1.0156562598 \times 10^{-9}$ & $1.0154658442 \times 10^{-9}$ \\
\hline 0.8 & $4.0974497948 \times 10^{-7}$ & $4.0965791893 \times 10^{-7}$ \\
\hline 0.9 & $1.6530326148 \times 10^{-4}$ & $1.6526400688 \times 10^{-4}$ \\
\hline 0.91 & $3.0120361268 \times 10^{-4}$ & $3.0113133218 \times 10^{-4}$ \\
\hline 0.92 & $5.4883742990 \times 10^{-4}$ & $5.4870434755 \times 10^{-4}$ \\
\hline 0.93 & $1.0000994228 \times 10^{-3}$ & $9.9985438896 \times 10^{-4}$ \\
\hline 0.94 & $1.8226172335 \times 10^{-3}$ & $1.8221659380 \times 10^{-3}$ \\
\hline 0.95 & $3.3229471186 \times 10^{-3}$ & $3.3221149126 \times 10^{-3}$ \\
\hline 0.96 & $6.0664843562 \times 10^{-3}$ & $6.0649430491 \times 10^{-3}$ \\
\hline 0.97 & $1.1125591069 \times 10^{-2}$ & $1.1122693539 \times 10^{-2}$ \\
\hline 0.98 & $2.0729215913 \times 10^{-2}$ & $2.0723473236 \times 10^{-2}$ \\
\hline 0.99 & $4.1128668075 \times 10^{-2}$ & $4.1114486364 \times 10^{-2}$ \\
\hline 0.995 & $6.3521002062 \times 10^{-2}$ & $6.3489058651 \times 10^{-2}$ \\
\hline 0.996 & $7.0878873876 \times 10^{-2}$ & $7.0840307461 \times 10^{-2}$ \\
\hline 0.997 & $8.0413553441 \times 10^{-2}$ & $8.0363176998 \times 10^{-2}$ \\
\hline 0.998 & $9.3908931099 \times 10^{-2}$ & $9.3834079185 \times 10^{-2}$ \\
\hline 0.999 & $1.1707300468 \times 10^{-1}$ & $1.1692354377 \times 10^{-1}$ \\
\hline
\end{tabular}

Table 8: Solution $y(x)$ for $\lambda=80, N=5, h=10^{-4}$.

\begin{tabular}{|c|c|c|}
\hline$x$ & $y(x)$ This article (Approximate Analytical) & $y(x)[5]$ (Numerical by Finite Difference) \\
\hline 0.1 & $2.6919498686 \times 10^{-33}$ & $2.6917305376 \times 10^{-33}$ \\
\hline 0.2 & $8.0234610791 \times 10^{-30}$ & $8.0234610791 \times 10^{-30}$ \\
\hline 0.3 & $2.3920966740 \times 10^{-26}$ & $2.3916183118 \times 10^{-26}$ \\
\hline 0.4 & $7.1307396863 \times 10^{-23}$ & $7.1288912518 \times 10^{-23}$ \\
\hline 0.5 & $2.1256435421 \times 10^{-19}$ & $2.1249666064 \times 10^{-19}$ \\
\hline 0.6 & $6.3364540945 \times 10^{-16}$ & $6.3340608222 \times 10^{-16}$ \\
\hline 0.7 & $1.8888703442 \times 10^{-12}$ & $1.8880450346 \times 10^{-12}$ \\
\hline 0.8 & $5.6306431392 \times 10^{-9}$ & $5.6278494204 \times 10^{-9}$ \\
\hline 0.9 & $1.6784711268 \times 10^{-5}$ & $1.6775389202 \times 10^{-5}$ \\
\hline 0.91 & $3.7355067448 \times 10^{-5}$ & $3.7334099563 \times 10^{-5}$ \\
\hline 0.92 & $8.3135292636 \times 10^{-5}$ & $8.3088135272 \times 10^{-5}$ \\
\hline 0.93 & $1.8502167037 \times 10^{-4}$ & $1.8491562270 \times 10^{-4}$ \\
\hline 0.94 & $4.1178073005 \times 10^{-4}$ & $4.1154226403 \times 10^{-4}$ \\
\hline 0.95 & $9.1651678613 \times 10^{-4}$ & $9.1598048794 \times 10^{-4}$ \\
\hline 0.96 & $2.0406494798 \times 10^{-3}$ & $2.0394421202 \times 10^{-3}$ \\
\hline 0.97 & $4.5515599019 \times 10^{-3}$ & $4.5488266110 \times 10^{-3}$ \\
\hline 0.98 & $1.0242709115 \times 10^{-2}$ & $1.0236341225 \times 10^{-2}$ \\
\hline 0.99 & $2.4212395085 \times 10^{-2}$ & $2.4194928841 \times 10^{-2}$ \\
\hline 0.995 & $4.0608245374 \times 10^{-2}$ & $4.0568761712 \times 10^{-2}$ \\
\hline 0.996 & $4.6079669175 \times 10^{-2}$ & $4.6031028714 \times 10^{-2}$ \\
\hline 0.997 & $5.3197491846 \times 10^{-2}$ & $5.3133088468 \times 10^{-2}$ \\
\hline 0.998 & $6.3304073785 \times 10^{-2}$ & $6.3207611241 \times 10^{-2}$ \\
\hline 0.999 & $8.0701566764 \times 10^{-2}$ & $8.0508119125 \times 10^{-2}$ \\
\hline
\end{tabular}


Table 9: Solution $y(x)$ for $\lambda=100, N=5, h=10^{-4}$.

\begin{tabular}{ccc}
\hline$x$ & $y(x)$ This article (Approximate Analytical) & $y(x)[5]$ (Numerical by Finite Difference) \\
\hline 0.1 & $3.2817751993 \times 10^{-41}$ & $3.2812814662 \times 10^{-41}$ \\
\hline 0.2 & $7.2285909322 \times 10^{-37}$ & $7.2266669708 \times 10^{-37}$ \\
\hline 0.3 & $1.5922031091 \times 10^{-32}$ & $1.5915951111 \times 10^{-32}$ \\
\hline 0.4 & $3.5070607321 \times 10^{-28}$ & $3.5053158087 \times 10^{-28}$ \\
\hline 0.5 & $7.7248153256 \times 10^{-24}$ & $7.7200783248 \times 10^{-24}$ \\
\hline 0.6 & $1.7015038054 \times 10^{-19}$ & $1.7002636165 \times 10^{-19}$ \\
\hline 0.7 & $3.7478115370 \times 10^{-15}$ & $3.7446464195 \times 10^{-15}$ \\
\hline 0.8 & $8.2551042624 \times 10^{-11}$ & $8.2471780674 \times 10^{-11}$ \\
\hline 0.9 & $1.8183077179 \times 10^{-6}$ & $1.8163516258 \times 10^{-6}$ \\
\hline 0.91 & $4.9426728499 \times 10^{-6}$ & $4.9372984970 \times 10^{-6}$ \\
\hline 0.92 & $1.3435578229 \times 10^{-5}$ & $1.3420813892 \times 10^{-5}$ \\
\hline 0.93 & $3.6521696929 \times 10^{-5}$ & $3.6481141045 \times 10^{-5}$ \\
\hline 0.94 & $9.9276441364 \times 10^{-5}$ & $9.9165050699 \times 10^{-5}$ \\
\hline 0.95 & $2.6986488685 \times 10^{-4}$ & $2.6955896307 \times 10^{-4}$ \\
\hline 0.96 & $7.3363994085 \times 10^{-4}$ & $7.3279961426 \times 10^{-4}$ \\
\hline 0.97 & $1.9956707542 \times 10^{-3}$ & $1.9933581879 \times 10^{-3}$ \\
\hline 0.98 & $5.4538456304 \times 10^{-3}$ & $5.4473878454 \times 10^{-3}$ \\
\hline 0.99 & $1.5460393744 \times 10^{-2}$ & $1.5440100274 \times 10^{-2}$ \\
\hline 0.995 & $2.8185450932 \times 10^{-2}$ & $2.8138301906 \times 10^{-2}$ \\
\hline 0.996 & $3.2514978714 \times 10^{-2}$ & $3.2454298524 \times 10^{-2}$ \\
\hline 0.997 & $3.8175308826 \times 10^{-2}$ & $3.8095308234 \times 10^{-2}$ \\
\hline 0.998 & $4.6244928533 \times 10^{-2}$ & $4.6125235801 \times 10^{-2}$ \\
\hline 0.999 & $6.0186796128 \times 10^{-2}$ & $5.9946471301 \times 10^{-2}$ \\
\hline
\end{tabular}

Table 10: Solution $y(x)$ for $\lambda=200,500, N=10, h=10^{-5}$.

\begin{tabular}{ccc}
\hline$x$ & $y(x)(\lambda=200)$ & $y(x)(\lambda=500)$ \\
\hline 0.1 & $1.3428368572 \times 10^{-80}$ & $2.9551064546 \times 10^{-198}$ \\
\hline 0.2 & $6.5149770621 \times 10^{-72}$ & $1.5321356773 \times 10^{-176}$ \\
\hline 0.3 & $3.1608401194 \times 10^{-63}$ & $7.9436723167 \times 10^{-155}$ \\
\hline 0.4 & $1.5335296142 \times 10^{-54}$ & $4.1185601775 \times 10^{-133}$ \\
\hline 0.5 & $7.4401519495 \times 10^{-46}$ & $2.1353521721 \times 10^{-111}$ \\
\hline 0.6 & $3.6097027745 \times 10^{-37}$ & $1.1071172211 \times 10^{-89}$ \\
\hline 0.7 & $1.7513021520 \times 10^{-28}$ & $5.7400767771 \times 10^{-68}$ \\
\hline 0.8 & $8.4967085082 \times 10^{-20}$ & $2.9760607799 \times 10^{-46}$ \\
\hline 0.9 & $4.1223072436 \times 10^{-11}$ & $1.5429998780 \times 10^{-24}$ \\
\hline 0.91 & $3.0459959480 \times 10^{-10}$ & $2.2900148637 \times 10^{-22}$ \\
\hline 0.92 & $2.2507034937 \times 10^{-9}$ & $3.3986834030 \times 10^{-20}$ \\
\hline 0.93 & $1.6630574377 \times 10^{-8}$ & $5.0440934063 \times 10^{-18}$ \\
\hline 0.94 & $1.2288424703 \times 10^{-7}$ & $7.4860983722 \times 10^{-16}$ \\
\hline 0.95 & $9.0799859559 \times 10^{-7}$ & $1.1110355087 \times 10^{-13}$ \\
\hline 0.96 & $6.7092528077 \times 10^{-6}$ & $1.6489228973 \times 10^{-11}$ \\
\hline 0.97 & $4.9575145051 \times 10^{-5}$ & $2.4472185630 \times 10^{-9}$ \\
\hline 0.98 & $3.6635374732 \times 10^{-4}$ & $3.6319943820 \times 10^{-7}$ \\
\hline 0.99 & $2.7234146882 \times 10^{-3}$ & $5.3904391730 \times 10^{-5}$ \\
\hline 0.995 & $7.7193683262 \times 10^{-3}$ & $6.5816087186 \times 10^{-4}$ \\
\hline 0.996 & $9.6771834475 \times 10^{-3}$ & $1.0893658752 \times 10^{-3}$ \\
\hline 0.997 & $1.2333583183 \times 10^{-2}$ & $1.8155829468 \times 10^{-3}$ \\
\hline 0.998 & $1.6226481832 \times 10^{-2}$ & $3.0877473301 \times 10^{-3}$ \\
\hline 0.999 & $2.3059106687 \times 10^{-2}$ & $5.6273164515 \times 10^{-3}$ \\
\hline & &
\end{tabular}




\section{Conclusion}

In this paper, we have proposed a new technique for finding an approximate analytical solution of the Troesch problem. Based on polynomial projection of the nonlinear term, and multistage implementation of Parker-Sochacki method, a highly accurate piecewise continuous solution of the Troesch problem, valid on the entire integration interval was obtained. For small and large values of the Troesch parameter $\lambda$, the technique produced accurate results when compared with existing results in the literature.

\section{References}

[1] E. S. Weibel. On the confinement of a plasma by magnetostatic fields. Physics of Fluids, 2(1):52-56, 1959.

[2] D. Gidaspow and B. S. Baker. A model for discharge of storage batteries. Journal of the Electrochemical Society, $120(8): 1004$ - 1010, 1973.

[3] V. S. Markin, A. A. Chernenko, Y. A. Chizmadehev, and Y. G. Chirkov. Aspects of the theory of gas porous electrodes, pages 22 - 33. in Fuel Cells: Their Electrochemical Kinetics. Consultants Bureau, New York, USA, 1966.

[4] S. M. Roberts and J. S. Shipman. On the closed form solution of troesch's problem. Journal of Computational Physics, 21(3):291 - 304, 1976.

[5] Helmi Temimi, Mohamed Ben-Romdhane, Ali R. Ansari, and Grigorii I. Shishkin. Finite difference numerical solution of troesch's problem on piecewise uniform shishkin mesh. Calcolo, 2016.

[6] Shih-Hsiang Chang. A variational iteration method for solving troesch's problem. Journal of Computational and Applied Mathematics, 234(10):3043 $3047,2010$.

[7] Xinlong Feng, Liquan Mei, and Guoliang He. An efficient algorithm for solving troesch's problem. Applied Mathematics and Computation, 189(1):500 $-507,2007$.

[8] Elias Deeba, S.A. Khuri, and Shishen Xie. An algorithm for solving boundary value problems. Journal of Computational Physics, 159(2):125 - 138, 2000.

[9] Hany N. Hassan and Magdy A. El-Tawil. An efficient analytic approach for solving two-point nonlinear boundary value problems by homotopy analysis method. Mathematical Methods in the Applied Sciences, 34(8):977-989, 2011.

[10] S. A. Khuri. A numerical algorithm for solving troesch's problem. International Journal of Computer Mathematics, 80(4):493-498, 2003.

[11] Mirmorandi S. H., Hosseinpour I., Ghanbarpour S., and Barari A. Application of an approximate analytical method to nonlinear troesch's problem. Applied Mathematical Sciences, 3(32):1579-1585, 2009.

[12] Gilberto González-Parra, Abraham J Arenas, and Lucas Jódar. Piecewise finite series solutions of seasonal diseases models using multistage adomian method. Communications in Nonlinear Science and Numerical Simulation, 14(11):3967-3977, 2009.

[13] M. Mossa Al-Sawalha, M.S.M. Noorani, and I. Hashim. On accuracy of adomian decomposition method for hyperchaotic rãglssler system. Chaos, Solitons \& Fractals, 40(4):1801 - 1807, 2009.

[14] A.K. Alomari, M.S.M. Noorani, and R. Nazar. Adaptation of homotopy analysis method for the numericâ€"analytic solution of chen system. Communications in Nonlinear Science and Numerical Simulation, 14(5):2336 - 2346, 2009.

[15] A K Alomari, M S M Noorani, and R Nazar. Homotopy approach for the hyperchaotic chen system. Physica Scripta, 81(4):7, 2010.

[16] Batiha B., M. S. M. Noorani, Hashim I., and Ismail E. S. The multistage variational iteration method for a class of nonlinear system of odes. Physica Scripta, 76:388-392, 2007.

[17] M.S.H. Chowdhury, I. Hashim, and S. Momani. The multistage homotopy-perturbation method: A powerful scheme for handling the lorenz system. Chaos, Solitons \& Fractals, 40(4):1929 - 1937, 2009.

[18] Do Y. and Jang B. Enhanced multistage differential transformation method: application to population models. Abstract and Applied Analysis, 2012(253890), 2012.

[19] D. C. Carothers, G. E. Parker, J. S. Sochacki, and P. G. Warne. Some properties of solutions to polynomial systems of differential equations. Electronic Journal of Differential Equations, 2005(40):1 - 17, 2005.

[20] P.G. Warne, D.A. Polignone Warne, J.S. Sochacki, G.E. Parker, and D.C. Carothers. Explicit a-priori error bounds and adaptive error control for approximation of nonlinear initial value differential systems. Computers \& Mathematics with Applications, 52(12):1695 - 1710, 2006.

[21] G. Edgar Parker and James S. Sochacki. Implementing the picard iteration. Neural, Parallel Sci. Comput., 4(1):97-112, March 1996.

[22] G. E. Parker and J. S. Sochacki. A Picard-Maclaurin theorem for initial value PDEs. Abstract and Applied Analysis, 5:47-63, 2000.

[23] Donald E. Knuth. The Art of Computer Programming, Volume 1 (3rd Ed.). Addison Wesley Longman Publishing Co., Inc., Redwood City, CA, USA, 1997.

[24] Amaury Pouly and Daniel S. Graca. Computational complexity of solving polynomial differential equations over unbounded domains. Theoretical Computer Science, 626:67 - 82, 2016.

[25] Martin Hermann and Masoud Saravi. Nonlinear Ordinary Differential Equations: Analytical Approximation and Numerical Methods. Springer India, 2016.

[26] Hector Vazquez-Leal, Yasir Khan, Guillermo Fernandez-Anaya, Agustin Herrera-May, Arturo Sarmiento-Reyes, Uriel Filobello-Nino, Victor-M. Jimenez-Fernandez, and Domitilo Pereyra-Diaz. A general solution for troesch's problem. Mathematical Problems in Engineering, Volume 2012(Article ID 208375):14 pages, 2012.

[27] M. Kubicek and V. Hlavacek. Solution of troesch's two-point boundary value problem by shooting technique. Journal of Computational Physics, 17(1):95 - 101, 1975.

[28] Youdong Lin, Joshua A. Enszer, and Mark A. Stadtherr. Enclosing all solutions of two-point boundary value problems for \{ODEs $\}$. Computers \& Chemical Engineering, 32(8):1714 - 1725, 2008.

[29] Utku Erdogan and Turgut Ozis. A smart nonstandard finite difference scheme for second order nonlinear boundary value problems. Journal of Computational Physics, 230(17):6464 - 6474, 2011. 